\title{
Nivel de actividad física y estado nutricional en una población pediátrica de un consultorio ambulatorio Asunción
}

\section{Level of physical activity and nutritional status in a pediatric population of an Asunción outpatient clinic}

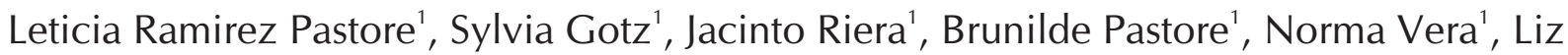 \\ Castaño', Victor Guillermo Sequera ${ }^{2}$
}

\section{RESUMEN}

Introducción: El descenso en el nivel de actividad física en los niños y adolescentes se asocia con mayores niveles de sobrepeso, obesidad y empeoramiento de la salud cardiovascular. Objetivo: Analizar los hábitos de práctica física, parámetros antropométricos y tiempo de pantalla (televisión, teléfonos móviles, tabletas) en una población escolar que acude a consulta pediátrica ambulatoria en Asunción durante el 2018. Materiales y Métodos: Estudio observacional, descriptivo con componente analítico, trasversal. El nivel de actividad física se valoró a través del cuestionario Physical Activity Questionnaire for Children (PAQ-C), con puntajes de 1 a 5 , siendo 5 la máxima actividad física. Se incorporó una pregunta sobre el número de horas al día frente a las pantallas. Las variables antropométricas evaluadas fueron el índice de masa corporal (IMC), Z-score del IMC, pliegue tricipital y circunferencia abdominal. Las variables se presentaron como media y desviación estándar. Los datos se analizaron las pruebas T-Student y Chi cuadrado. El nivel de significancia establecido fue $<0,05$. Resultados: Fueron evaluados 636 escolares, $54 \%$ de sexo masculino, con edad media de 10 años, un 11,3\% presentó obesidad y un 22,2\% sobrepeso. La actividad física medida por PAQ-C obtuvo de media 2,93 $\pm 0,65$ puntos. El promedio de tiempo de pantalla fue de 3,53 $\pm 2,33$ horas. En función del sexo no se encontraron diferencias significativas para la edad, tampoco para los parámetros antropométricos, ni horas de pantalla. La división en terciles de los valores de actividad física mostró diferencias significativas con el IMC, Z-score del IMC y pliegue tricipital, no así con la circunferencia abdominal $(\mathrm{p}=0,089)$. Por otro lado, los niños menos

\begin{abstract}
Introduction: The decrease in the level of physical activity in children and adolescents is associated with higher levels of being overweight, obesity and worsening of cardiovascular health. Objective: To analyze physical activity habits, anthropometric parameters and screen time (television, mobile phones, tablets) in a school-aged population who presented for pediatric ambulatory consultation in Asunción during 2018. Materials and Methods: This was an observational cross-sectional and descriptive study with an analytical component. The level of physical activity was assessed through the Physical Activity Questionnaire for Children (PAQ-C), with scores ranging from 1 to 5 , with 5 being the maximum physical activity. A question about the number of hours per day in front of screens was incorporated. The anthropometric variables evaluated were body mass index (BMI), BMI Zscore, triceps skin fold and abdominal circumference. The variables were presented as mean and standard deviations. The data were analyzed by the T-Student and Chi square tests. The level of significance established was $<0.05$. Results: 636 schoolchildren were evaluated, $54 \%$ were male, with a mean age of 10 years. $11.3 \%$ were obese and $22.2 \%$ overweight. The physical activity measured by PAQ-C averaged $2.93 \pm 0.65$ points. The average screen time was $3.53 \pm 2.33$ hours. When adjusted for gender, no significant differences were found for age, nor for anthropometric parameters, nor screen hours. The tertile division of the physical activity values showed significant differences with the BMI, Z-score of the BMI and triceps skin fold, but not with the abdominal circumference ( $\mathrm{p}=$ 0.089 ). On the other hand, the least active children are those who said they spend more time in front of screens ( $p$ $=0.006)$. Conclusion: Lower levels of physical activity are reported by those children who spend more time in front
\end{abstract}

${ }^{1}$ Centro Pediátrico Integral Riera S.R.L. Asunción, Paraguay.

${ }^{2}$ Ministerio de Salud Pública y Bienestar Social, Dirección de Vigilancia de la Salud. Asunción. Paraguay

Correspondencia: Leticia Ramirez Pastore Correo: letty.ramirez@hotmail.com

Conflicto de interés: Los autores declaran no poseer conflicto de interés

Recibido: 15/11/2019 Aceptado: 6/02/2020

DOI: https://doi.org/10.31698/ped.47012020003

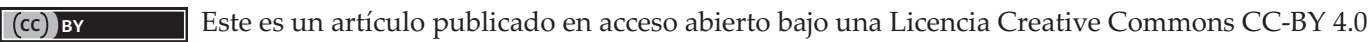


activos son los que manifestaron pasar más tiempo frente a las pantallas $(\mathrm{p}=0,006)$. Conclusión: Los menores niveles de actividad física son reportados por aquellos niños que pasan más tiempo frente a las pantallas, que presentan un mayor índice de masa corporal y Z-score IMC, así como un mayor pliegue tricipital.

Palabras clave: Actividad física, sedentarismo, escolares, obesidad, sobrepeso.

\section{INTRODUCCIÓN}

El sedentarismo y la disminución en los niveles de actividad física (AF) se han convertido en un verdadero problema de salud pública que también afecta a los niños de todas las edades, con un aumento marcado en los últimos años ${ }^{(1)}$. Este descenso en el nivel de actividad física se asocia con mayores niveles de sobrepeso y obesidad ${ }^{(2)}$ y un empeoramiento de la salud cardiovascular de niños y jóvenes ${ }^{(3)}$. La Organización Mundial de la Salud (OMS) recomienda un mínimo de 60 minutos diarios de ejercicio físico moderado o vigoroso en niños de 5 a 18 años $^{(4)}$, ya que incrementar el ejercicio físico ha demostrado mejora en parámetros saludables como perfil lipídico ${ }^{(5)}$, resistencia a la insulina ${ }^{(6)}$, masa $\operatorname{grasa}^{(7)}, \mathrm{y}$ otros datos asociados al síndrome metabólico ${ }^{(8)}$.

Otro factor importante que se asocia directamente a la disminución de la AF es la gran cantidad de tiempo que pasan los niños frente a las pantallas ${ }^{(9)}$, tales como teléfonos móviles, tabletas, computadoras, televisión, videojuegos, etc.

Es, por tanto, necesario e importante dar una respuesta desde el punto de vista de la salud pública a la preocupación actual relacionada con la inactividad física a través de la vigilancia de los niveles de AF utilizando un protocolo estandarizado. El método más fácil y de menor costo para evaluar los niveles de AF es a través de cuestionarios, instrumento que debe ser apropiado, válido y fiable, incluyendo varios componentes como la frecuencia, intensidad y duración de la actividad física, lo cual presenta un reto complejo. Es por ello que la OMS desarrolló un cuestionario de of screens, have a higher body mass index and BMI Zscore, as well as a greater triceps skin fold.

Keywords: Physical activity, sedentary lifestyle, school children, obesity, overweight.

actividad física "PAQ", por sus siglas en inglés, Physical Activity Questionnaire, y en este caso particular, el "PAQ-C", o cuestionario de actividad física en niños (Children), instrumento utilizado en el presente estudio para medir el nivel de actividad física en los niños evaluados ${ }^{10}$.

El objetivo de este estudio fue analizar asociación entre los hábitos de actividad física, parámetros antropométricos y tiempo de pantalla en una población pediátrica que acudió a consulta pediátrica ambulatoria en Asunción, Paraguay.

\section{MATERIALES Y MÉTODOS}

Estudio observacional, descriptivo con componente analítico, de corte trasversal, llevado a cabo en un consultorio pediátrico privado.

Fueron evaluados pacientes de ambos sexos, de 5 a 19 años edad, que acudieron a consulta de niño sano y chequeo médico previo al inicio de clases, de enero a marzo de 2018.

El nivel de actividad física se valoró a través del cuestionario PAQ-C, en su versión validada y adaptada al español en 2009 por Martínez Gómez y colaboradores $^{10}$. El cuestionario valora la práctica moderada a vigorosa, realizada en los últimos 7 días de una semana habitual, a través de 10 preguntas sobre el tipo y frecuencia de actividades que se realizan. El cuestionario cuenta con 10 preguntas, las 9 primeras se utilizan para calcular la puntuación final y la pregunta restante sirve para identificar si el niño estuvo enfermo o existió alguna circunstancia 
que le impidió realizar AF en esa semana de manera rutinaria. De las respuestas se obtiene una puntuación de 1 a 5, indicando una mayor puntuación una mayor actividad. En función de la puntuación final, se decidió clasificar a los pacientes en terciles correspondientes al nivel de actividad física: bajo, medio y alto, para luego realizar el análisis.

Dicho cuestionario incorporó una pregunta sobre el número de horas que pasaban al día frente a las pantallas (TV, computadora, consola, tableta, teléfono móvil, otros). El cuestionario fue completado por el padre o la madre en colaboración directa con el hijo.

Las medidas antropométricas fueron tomadas por un profesional médico. Se midió el peso y la altura con una balanza electrónica de precisión (SECA) con cartabón incluido (metodología de Frankfurt). A partir de estos datos, se calculó el índice de masa corporal (IMC) como el peso divido por la altura al cuadrado $\left(\mathrm{Kg} / \mathrm{m}^{2}\right)$. Para determinar el estado nutricional fueron utilizados los criterios diagnósticos de la OMS y fue utilizado el software Anthro Plus para el cálculo del puntaje Z del $\mathrm{IMC}^{(1,1,2)}$. La circunferencia abdominal (CA) se midió con cinta métrica no distensible de fijación automática (SECA), medido al final de la espiración por sobre el reborde de la cresta iliaca, pasando por el ombligo. Para la medición del pliegue tricipital se utilizó el plicómetro de Jamar.

Para el análisis de datos se utilizó el programa estadístico STATA v15. Para determinar la normalidad en la distribución de las variables se utilizó la prueba de Kalmogorov-Smirmov. Las variables cuantitativas se presentaron como media y desviación estándar Las cualitativas como porcentajes. Los datos se analizaron según las pruebas de $t$ de Student para las variables cuantitativas y Chi cuadrado para las cualitativas. El nivel de significancia se estableció en $<0,05$.

\section{RESULTADOS}

Fueron analizados datos de 636 pacientes, 54,4\% de sexo masculino, con una edad promedio de $10 \pm 4$ años.
El valor promedio de índice de masa corporal fue de $18,82 \mathrm{~kg} / \mathrm{m}^{2}, Z$-score del índice de masa corporal 0,39 , pliegue tricipital $13,93 \mathrm{~mm}$ y circunferencia abdominal $65,5 \mathrm{~cm}$.

El promedio de tiempo de pantalla fue de 3,53 $\pm 2,33$ horas, con valores promedio mayores a 3 horas en ambos sexos.

La actividad física, medida según el puntaje de PAQC con valores de 1 a 5, obtuvo una media de 2,93 \pm 0,65 puntos. En niños el promedio fue de $3,03 \pm 0,67 \mathrm{y}$ en niñas $2,81 \pm 0,6$ puntos (Tabla 1 ).

Para el análisis, el puntaje PAQ-C se dividió en terciles, resultando el $36,69 \%$ de los pacientes en tercil bajo, 33,70\% en tercil medio y $29,61 \%$ en el más alto.

La edad, parámetros antropométricos y horas de pantalla al día se resumen en las tablas 2 y 3 , los cuales quedaron divididos en función del sexo y el nivel de actividad física.

En función del sexo, no se encontraron diferencias significativas para la edad, así como tampoco para IMC; Z-score del IMC; pliegue tricipital, circunferencia abdominal ni horas de pantalla. (Tabla 2)

Tabla 1. Características de la población ( $\mathrm{n}=636)$.

\begin{tabular}{|c|c|}
\hline & $\%$ Media (+DE) \\
\hline Edad, años & $10,5 \pm 3,7$ \\
\hline \multicolumn{2}{|l|}{ Sexo, $\%$} \\
\hline Masculino & $54,4 \%$ \\
\hline Femenino & $45,6 \%$ \\
\hline IMC, Z-score & $0,39 \pm 1,05$ \\
\hline Pliegue tricipital, $\mathrm{mm}$ & $13,93+7,12$ \\
\hline Circunferencia abdominal, $\mathrm{cm}$ & $65,5+11,14$ \\
\hline \multicolumn{2}{|l|}{ Estado nutricional } \\
\hline Bajo peso & $9,3 \%$ \\
\hline Peso adecuado & $57,2 \%$ \\
\hline Sobrepeso & $22,2 \%$ \\
\hline Obesidad & $11,3 \%$ \\
\hline PAQ-C & $2,93 \pm 0,65$ \\
\hline Masculino & $3,03 \pm 0,67$ \\
\hline Femenino & $2,81+0,6$ \\
\hline \multicolumn{2}{|l|}{ Nivel de Actividad Física } \\
\hline Bajo & $36,7 \%$ \\
\hline Medio & $33,7 \%$ \\
\hline Alto & $29,6 \%$ \\
\hline Tiempo de Pantalla, horas & $3,53 \pm 2,33$ \\
\hline Masculino & $3,56 \pm 2,29$ \\
\hline Femenino & $3,48 \pm 2,37$ \\
\hline
\end{tabular}


Tabla 2. Características de la muestra en función del sexo.

\begin{tabular}{lcccc}
\cline { 2 - 5 } & IOTAL & $\begin{array}{c}\text { Femenino } \\
(\mathbf{n = 2 9 0 )}\end{array}$ & $\begin{array}{c}\text { SEXO } \\
\text { Masculino } \\
(\mathbf{n = 3 4 6})\end{array}$ & $\begin{array}{c}\mathbf{p} \\
\text { valor* }\end{array}$ \\
\hline Edad, años & $10,46 \pm 3,74$ & $10,61 \pm 3,73$ & $10,34 \pm 3,75$ & 0,18 \\
IMC & $18,82 \pm 3,62$ & $18,87 \pm 3,5$ & $18,78 \pm 3,73$ & 0,37 \\
IMC, Z-score & $0,39 \pm 1,05$ & $0,46 \pm 1,06$ & $0,57 \pm 1,31$ & 0,11 \\
\hline P. tricipital, $m m$ & $13,93 \pm 7,12$ & $14,14 \pm 5,77$ & $13,76 \pm 8,11$ & 0,26 \\
CA, $c m$ & $65,5 \pm 11,14$ & $64,35 \pm 10,37$ & $66,46 \pm 11,68$ & 0,052 \\
Horas Pantalla & $3,53 \pm 2,33$ & $3,48 \pm 2,37$ & $3,56 \pm 2,29$ & 0,34 \\
\hline *Test T-student & & & & \\
\hline
\end{tabular}

*Test T-student

IMC: Indice de masa corporal, P. Tricipital, CA: Circunferencia abdominal.

La división en terciles de actividad física mostró diferencias significativas para los valores de índice de masa corporal $(p=0,018), Z$-score del IMC $(p=0,04)$ y pliegue tricipital $(\mathrm{p}<0,001)$, no así circunferencia abdominal $(\mathrm{p}=0,089)$, donde la poca diferencia no arroja significancia estadística. Por otro lado, son los niños menos activos los que manifiestan pasar más tiempo frente a las pantallas $(\mathrm{p}=0,006)$. (Tabla 3$)$.

Tabla 3. Características de la muestra en función al nivel de Actividad Física.

\begin{tabular}{|c|c|c|c|c|c|}
\hline & \multirow{2}{*}{$\begin{array}{l}\underline{\text { TOTAL }} \\
(n=636)\end{array}$} & \multicolumn{4}{|c|}{ ACTIVIDAD FíSICA (terciles) } \\
\hline & & $\begin{array}{c}\text { Bajo } \\
(n=233)\end{array}$ & $\begin{array}{c}\text { Medio } \\
(\mathrm{n}=214)\end{array}$ & $\begin{array}{c}\text { Alto } \\
(n=188)\end{array}$ & $\begin{array}{c}\mathbf{p} \\
\text { valor* }\end{array}$ \\
\hline Edad, años & $10,46 \pm 3,74$ & $10,77 \pm 3,93$ & $10,09 \pm 3,64$ & $10,49 \pm 3,6$ & 0,355 \\
\hline IMC & $18,82 \pm 3,62$ & $19,41 \pm 3,62$ & $18,74 \underline{ \pm} 3,51$ & $18,19 \pm 3,23$ & 0,018 \\
\hline IMC, Z-score & $0,39 \pm 1,05$ & $0,39 \pm 1,05$ & $0,59 \pm 1,2$ & $0,3 \pm 1,09$ & 0,04 \\
\hline P. tricipital, $m m$ & $13,93 \pm 7,12$ & $13,93 \pm 7,12$ & $14,06+6,38$ & $12,98 \pm 0,04$ & $<0,001$ \\
\hline $\mathrm{CA}, \mathrm{cm}$ & $65,5 \pm 11,14$ & $65,5 \pm 11,92$ & $65,68 \pm 10,96$ & $64,12 \pm 10,24$ & 0,089 \\
\hline Horas Pantalla & $3,53 \pm 2,33$ & $4,07 \pm 2,55$ & $3,35+2,08$ & $3,06+2,19$ & 0,006 \\
\hline
\end{tabular}

*Test T-student

IMC: Indice de masa corporal, P. Tricipital, CA: Circunferencia abdominal.

\section{DISCUSIÓN}

Es el primer estudio en nuestro país donde se aplica el cuestionario PAQ-C, instrumento desarrollado por la OMS, para determinar el nivel de actividad física en niños. Hay reportes de estudios previos realizados en el país con un cuestionario diferente ${ }^{(13)}$. Los resultados del presente estudio no encontraron diferencias en cuanto al sexo en ninguno de los parámetros antropométricos evaluados: índice de masa corporal, Z-score del índice de masa corporal, pliegue tricipital, circunferencia abdominal, coincidiendo con estudios realizados previamente con pacientes de edades similares, tanto en nuestro país $^{(13)}$ como en España ${ }^{(14)}$.

Por otro lado, no se encontró diferencias significativas en cuanto a sexo y tiempo de pantalla, donde varios estudios señalan que las niñas pasan menos tiempo frente a la pantalla que los niños ${ }^{(14,15)}$.
Este estudio reveló que los niños y niñas menos activos son los que tienen valores más altos de IMC, Z-score de IMC y pliegue tricipital, coincidiendo con otros estudios ${ }^{(16)}$ donde se encontró una asociación inversa entre la práctica de actividad física y el porcentaje graso. En relación a la circunferencia abdominal, a menor actividad física, mayor circunferencia abdominal, sin embargo dicha diferencia no resultó estadísticamente significativa.

Por otro lado, y como se demuestra en este estudio, se encontró una relación inversa entre el tiempo de pantalla y los niveles de actividad física, coincidiendo con estudios de niños y niñas de la misma edad ${ }^{(9)}$. Por tanto, coincidiendo con la literatura ${ }^{(17)}$, niños con peso adecuado, son físicamente más activos y con menos hábitos sedentarios.

Este trabajo no establece causalidad entre los niveles de actividad física y tiempo de pantalla en relación al 
sobrepeso u obesidad, pero si muestra una asociación significativa y similar a otros trabajos realizados y en país y la región ${ }^{(13,14,18)}$.

Las limitaciones que presenta el estudio incluyen los sesgos propios de una metodología basada en cuestionario como el sesgo de memoria y de información y la no utilización del acelerómetro como gold standard de actividad física. Además, la población corresponde a un consultorio pediátrico privado, por lo que los datos no pueden generalizarse

\section{REFERENCIAS}

1. Blair, SN. Physical inactivity: The biggest public health problem in the 21st century. British Journal of Sports Medicine. 2009;43(1):1-2.

2. Herman KM, Sabiston CM, Mathieu ME, Tremblay A, Paradis G. Correlates of sedentary behavior in 8 to 10 year old children at elevated risk for obesity. Applied psysiology, nutrition and metabolism. 2015;40(1): 10-19.

3. Andersen LB, Bugge A, Dencker M, Eiberg S, El-Naaman $B$. The association between physical activity, physical fitness and development of metabolic disorders. International Journal of Pediatric Obesity. 2011; 6(Supll 1):29-34.

4. World Health Organization. Good Recommendations on Physical Activity for Health. Geneva, WHO Press; 2010.

5. Llorente-Cantanero FJ, Perez-Navero JL, Benitez-Sillero JD, Muñoz-Villanueva MC, Gill-Campos M. Evaluation of metabolic risk in prepuberal girls versus boys in relation to fitness and physical activity. Gender Medicine. 2012; 9(6):436-444.

6. Berman LJ, Weigensberg MJ, Spruijt-Metz D. Physical activity is related to insulin sensitivity in children and adolescents, independent of adiposity: a review of the literature. Diabetes Metab Res Rev. 2012;28(5):395-408.

7. Pahkala K, Hernelahti M, Heinonen OJ, Raittinen P, Hakanen M, Lagtrom H. Body mass index, fitness and physical activity from childhood through adolescence. British Journal of Sports Medicina. 2013; 47(2):71-77.

8. Christodoulos AD, Douda HT, Tokmakidis SP. Cardiorespiratory Fitness, Metabolic Risk and Inflammation in Children. International Journal of Pediatrics. 2012; 2012:270515. DOI:10.1155/2012/270515. a nivel país, pero orientan acerca de la problemática en torno a la inactividad física y sedentarismo a tempranas edades.

\section{CONCLUSIÓN}

Los menores niveles de actividad física son reportados por aquellos niños que pasan más tiempo frente a las pantallas, que presentan un mayor índice de masa corporal y mayor pliegue tricipital, es decir, una composición corporal menos saludable.

9. Ariscado D, Muros JJ, Zabala M, Dalmau JM. Factors associated with low adherence to a Mediterranean diet in healthy children in northern Spain. Appetite. 2014; 80:28-34.

10. Martínez-Gómez D, Martínez-De Haro V, Pozo T, Welk GJ, Villagra A, Calle ME. Reliability and validity of the PAQ-A questionnaire to assess physical activity in Spanish adolescents. Revista Española de Salud Pública, 2009; 83(3), 427-439.

11. World Health Organization. OMS Anthro (versión 3.2.2, enero de 2011) y macros. Software. OMS; 2011. Disponible en: https://www.who.int/childgrowth/software/es/

12. World Health Organization. Patrones de crecimiento infantil Internet. OMS; 2011. Disponible en: https://www.who.int/childgrowth/standards/es/

13. Ruiz S, Mesquita M, Sánchez S. Actividad física y estado nutricional en adolescentes de 9 a 15 años de una institución de enseñanza de San Lorenzo, Paraguay. Pediatr (Asunción). 2017;44(2):111-116.

14. Muros JJ, Zabala M, Oliveras-López MJ. Results of a 7week school-based physical activity and nutrition pilot program on health-related parameters in primary school children in southern Spain. Pediatric Exercise Science, 2013; 25(2):248-261.

15. Le Blanc AG, Katzmarzyk PT, Barreira TV, Broyles ST, Chaput JP, Church TS, et al. Correlates of total sedentary time and screen time in 9-11 year-old children around the world: The international study of childhood obesity, lifestyle and the environment. PLoS One, 2015; 10(6):e0129622.

16. Blaes A, Baquet G, Fabre C, Van Praagh E, Berthoin S. Is there any relationship between physical activity level and 
patterns and physical performance in children?. Int J Behav Nutr Phys Act. 2011; 8:122.

17. Shi X, Tubb L, Fingers ST, Chen S, Caffrey JL. Associations of physical activity and dietary behaviors with children's health and academic problems. J Sch Health. 2013; 83(1):1-7.
18. Muros JJ, Cofre-Bolados C, Salvador Perez S, CastroSanchez M, Valdivia-Moral P, Perez-Cortés AJ. Relación entre nivel de actividad física y composición corporal en escolares de Santiago (Chile). Journal of Sport and Health Research. 2016;8(1):65-74. 